\title{
Skeletal complications in
}

\section{mucopolysaccharidosis VI patients: Case reports}

\author{
Paula Garcia ${ }^{\mathrm{a}, *}$, Sérgio B. Sousa ${ }^{\mathrm{b}}$, Tah Pu Ling ${ }^{\mathrm{c}}$, Mário Conceição ${ }^{\mathrm{c}}$, Jorge Seabra $^{\mathrm{c}}$, \\ Klane K. White ${ }^{\mathrm{d}}$ and Luisa Diogo ${ }^{\mathrm{a}}$ \\ ${ }^{a}$ Unidade de Doenças Metabólicas, Hospital Pediátrico de Coimbra, Coimbra, Portugal \\ ${ }^{\mathrm{b}}$ Serviço de Genética Médica, Hospital Pediátrico de Coimbra, Coimbra, Portugal \\ ${ }^{\mathrm{c}}$ Serviço de Ortopedia, Hospital Pediátrico de Coimbra, Coimbra, Portugal \\ ${ }^{\mathrm{d}}$ Children's Hospital and Regional Medical Center, Department of Orthopedic Surgery, Seattle, WA, USA
}

Accepted for publication 21 January 2010

\begin{abstract}
Mucopolysaccharidosis (MPS) VI is an inheritable lysosomal storage disorder that is often associated with severe orthopedic problems such as hip dysplasia, spinal deformities, and deformities in the skull, knees and hands. We describe the progression and management of three MPS VI cases with focus on their orthopedic problems.
\end{abstract}

Keywords: Bone, mucopolysaccharidosis VI, orthopedics, skeleton

\section{Introduction}

Mucopolysaccharidosis VI (Maroteaux-Lamy syndrome, MPS VI) is a lysosomal storage disorder caused by mutations in the $\mathrm{N}$-acetylgalactosamine-4-sulfatase (arylsulfatase $\mathrm{B}, A R S B$ ) gene, resulting in the accumulation of glycosaminoglycans (GAGs) in cells and tissues all over the body [13]. Disease onset and rate of progression are variable, producing a spectrum of systemic clinical manifestations with significant functional impairment [7]. The skeleton is generally one of the most severely affected organs, causing severe morbidity. MPS VI patients typically show dysostosis multiplex, a specific radiologic expression involving the skull, thorax, pelvis, hands and spine [12,13]. Complications that result from skeletal disease include disproportionate short stature, kyphoscoliosis, joint ab-

* Address for correspondence: Paula Garcia, Unidade de Doenças Metabólicas, Hospital Pediátrico de Coimbra, Coimbra, Portugal. Tel.: +351 239480606; Fax: +351 717116; E-mail: pg@chc.minsaude.pt. normalities (stiffness and flexion contractures of elbows, shoulders, hips, knees, and fingers), spinal cord or nerve root compression [7,12].

We describe three unrelated Portuguese MPS VI patients, focusing on their most important skeletal problems, in order to illustrate the wide spectrum of possible manifestations and to discuss the individual management of each case. All patients described are receiving enzyme replacement therapy (ERT) with recombinant human ARSB (galsulfase, Naglazyme ${ }^{\circledR}$ Biomarin Pharmaceutical Inc., Novato, CA, USA), which has recently become available for MPS VI [8].

\section{Case reports}

\subsection{Patient 1}

\subsubsection{Description}

Patient 1 is a 9.4 year-old boy, second child of healthy parents, without known consanguinity. At 6 months of age, his parents noticed a lumbar gibbus deformity and umbilical and bilateral inguinal hernias that had 
progressed over time. At the age of 1 year, he was referred to a surgical and orthopedic outpatient clinic, and he underwent surgical correction of the hernias. Orthopedic evaluation revealed anterior hypoplasia of the L2-L3 vertebral bodies and bilateral hip dysplasia with left hip luxation. At 1.5 years of age, he was surgically treated using a single-stage bilateral procedure that consisted of open reduction of the left hip, modified innominate Salter osteotomy and proximal femoral varus osteotomy. There was a good functional outcome after surgery.

At 3 years of age, bilateral corneal opacity was detected and the boy was referred to the Metabolic Unit with the clinical suspicion of MPS. By that time, he had coarse facial features, hepatosplenomegaly, mild conductive hearing loss, heart murmur, mitral and aortic valve dysplasia with associated insufficiency, and normal intellect. The diagnosis of MPS VI was made and a homozygous mutation c. $1143-8 \mathrm{~T}>\mathrm{G}$ (IVS5-8T $>\mathrm{G})$ at the $A R S B$ gene was identified, which is a common mutation in Brazilian patients [15] and in our Metabolic Unit in Hospital Pediátrico de Coimbra, Coimbra, Portugal (personal observation).

The patient's height was above the 95 th percentile at the age of 1.6 years, dropped to the 5 th percentile by the age of 4 years, and is presently well below the 5 th percentile at 9.4 years of age (corresponding with the 50 th percentile for children of 4.5 years). The thoracolumbar kyphosis has remained stable and the boy developed a mild scoliosis. He also developed a pectus carinatum deformity and joint stiffness and contractures, especially at the shoulders, elbows, wrists, hands, and knees. The main issue in this patient has been the progression of hip disease. Besides the related hip contracture and walking difficulties, the patient has been complaining of lateral intermittent hip pain, mainly on the right side, for the last 6 months. Figure 1 shows skeletal follow-up radiographs that were made in the last 8 years.

Weekly infusions of ERT with $1 \mathrm{mg} / \mathrm{kg}$ of galsulfase was started at 7.3 years. ERT was well tolerated, without reported adverse effects. The liver and spleen returned to normal size and, generally, joint mobility improved.

\subsubsection{Discussion}

This case describes a child with MPS VI, who presents at a very young age with classic skeletal findings. The presence of a gibbus deformity due to anterior hypoplasia of the vertebral body, particularly at this age, is very suggestive of MPS, and warrants fur- ther diagnostic evaluation. Lumbar gibbus deformity is the single most common physical finding leading to the diagnosis of MPS [2,5]. Hip dysplasia is also often apparent in these children. Surgical containment of the hips in MPS VI has not been previously described. Hip morphology seen on radiograph for this child at nine years of age was much improved. Note that the hips are located and round, despite the presence of epiphyseal dysplasia (which has the appearance of femoral head necrosis). This is in contrast with the untreated hips in Case 2.

\subsection{Patient 2}

\subsubsection{Description}

This 9.5 year-old boy is the only child of healthy non-consanguineous parents with irrelevant family and perinatal history. At 13 months, the parents noticed a lumbar gibbus deformity and later, their concerns increased as he did not walk independently at 16 months old. He was sent to the Metabolic Unit with the clinical suspicion of MPS, based on radiographic abnormalities and coarse facies. A diagnosis of MPS VI was made at 1.6 years. Mutation analysis revealed compound heterozygosity for the mutations c.149T $>$ A and c. $1143-8 \mathrm{~T}>\mathrm{G}$ (IVS5-8T $>\mathrm{G}$ ) of the ARSB gene.

Multi-organ evaluation disclosed corneal opacity without glaucoma, hepatosplenomegaly and cardiac valvular dysplasia, mainly at the mitral level, controlled with lisinopril and furosemide. The boy had also developed macrocephaly and short stature. His height was at the 50th percentile at 3.6 years but is presently below the 5 th percentile.

Radiological assessments revealed typical dysostosis multiplex. In the spine, the boy has mild cervical bone abnormalities and anterior hypoplasia of the L1L2 vertebral bodies. Bracing was used between 4.5 and 7 years. His thoraco-lumbar kyphosis has stabilized $\left(\sim 40^{\circ}\right)$, without significant scoliosis. He has mild contractures at the shoulders, wrists, hands, elbows, ankles and knees. This patient's main skeletal problems are the degeneration and contracture of the hips and the progressive, symmetric genu valgum (knock knees), that have affected the way he walked. At 8.1 years, the boy underwent bilateral lower femoral and proximal tibial medial hemiepiphysiodesis. The surgical procedure was uneventful and walking functionality recovered. Figure 2 illustrates some skeletal features of the patient at follow-up.

ERT with galsulfase $(1 \mathrm{mg} / \mathrm{kg} /$ week $)$ was started at the age of 7.3 years. As in case 1 , the infusions were 


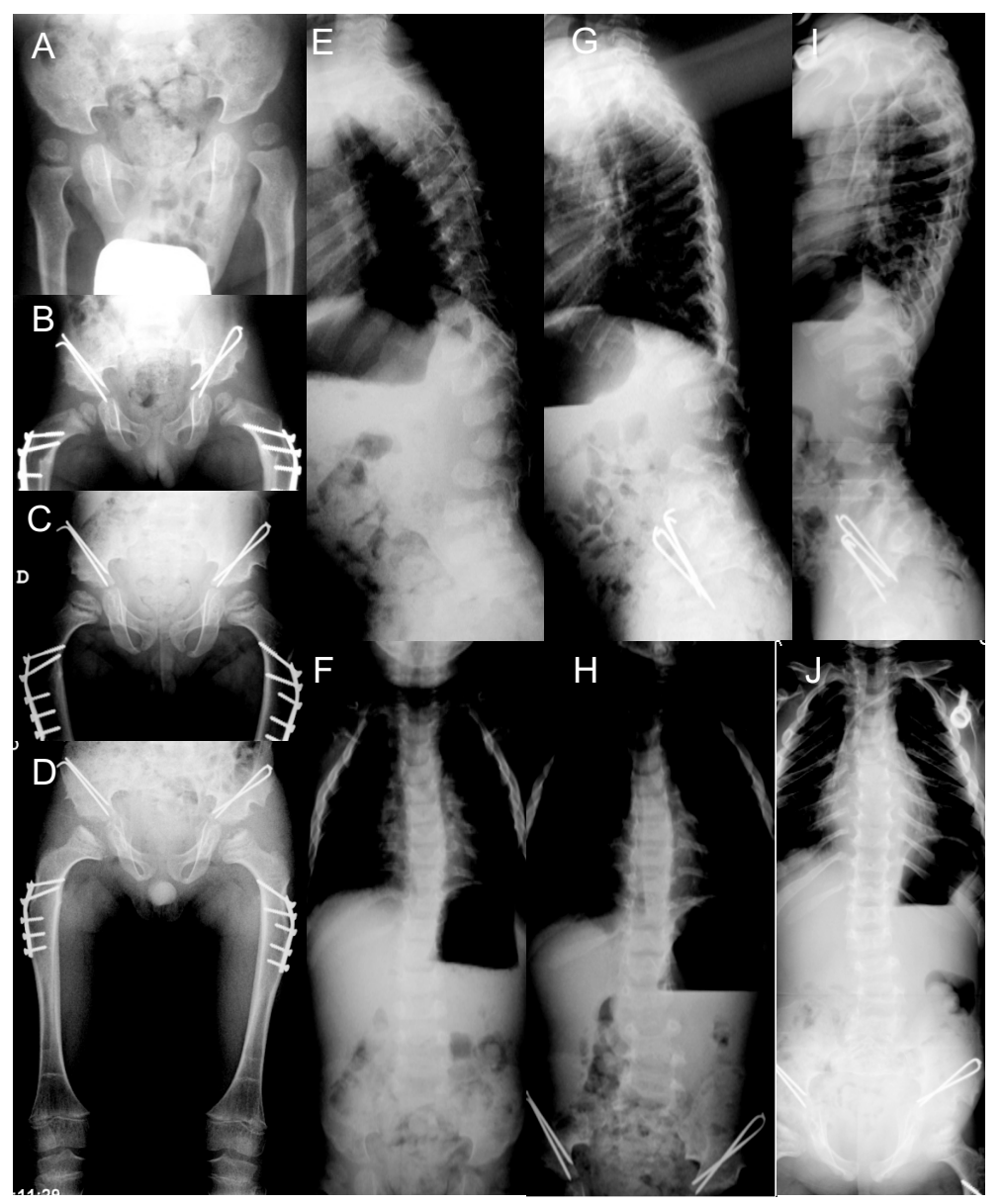

Fig. 1. X-ray films of Patient 1: hip at 11 months (A), 1.11 years (B), 5.2 years (C) and 8.10 years (D); spine at 11 months (E, F), 4.3 years (G, H) and 8.10 years (I, J). Notice the hip subluxation, surgical intervention and the progression of the femoral head abnormalities (A-D). There is L3 (and L2) severe hypoplasia. In the last years, the kyphosis has been stable but he developed a mild lumbar scoliosis (J). The cervico-diaphyseal angle before surgery was $170^{\circ}(\mathrm{A})$ and after surgery $135^{\circ}(\mathrm{B}, \mathrm{C}, \mathrm{D})$.

well tolerated, without reported adverse effects. The liver and spleen returned to normal sizes and joint mobility generally improved.

\subsubsection{Discussion}

The central focus of this case is the surgical care of the genu valgum deformity. Medial hemiepiphysiodesis has been shown to be quite successful in children with Hurler syndrome (MPS IH) after hematopoietic stem cell transplant [14]. The implants shown here utilize a tension band plate and screws and represent a fairly new device in the care of angular deformities in children [17]. "Guided growth" or "growth modulation" as originally described by Walter Blount in the 1940's, utilized staples to this end [3]. Failure of staples was common in MPS (as in other conditions), due to back out of the staples, often requiring revision surgery.
The tension band plates offer hope for fewer implant related complications, and have had a very good track record in other skeletal dysplasias. Because this technique relies on growth to be effective, patients must have at minimum one year of growth remaining in order to be considered candidates for this procedure. Some orthopedists, unfamiliar with MPS disorders, may wait too long for medial hemi-epiphysiodesis, assuming that MPS patients mature at the same age as uninvolved patients. However, because growth may terminate at an early age, surgical intervention that relies on continued growth should not be delayed.

\subsection{Patient 3}

This 2.8 year-old boy was born from non-consanguineous healthy parents. At 5 months of age, a lum- 


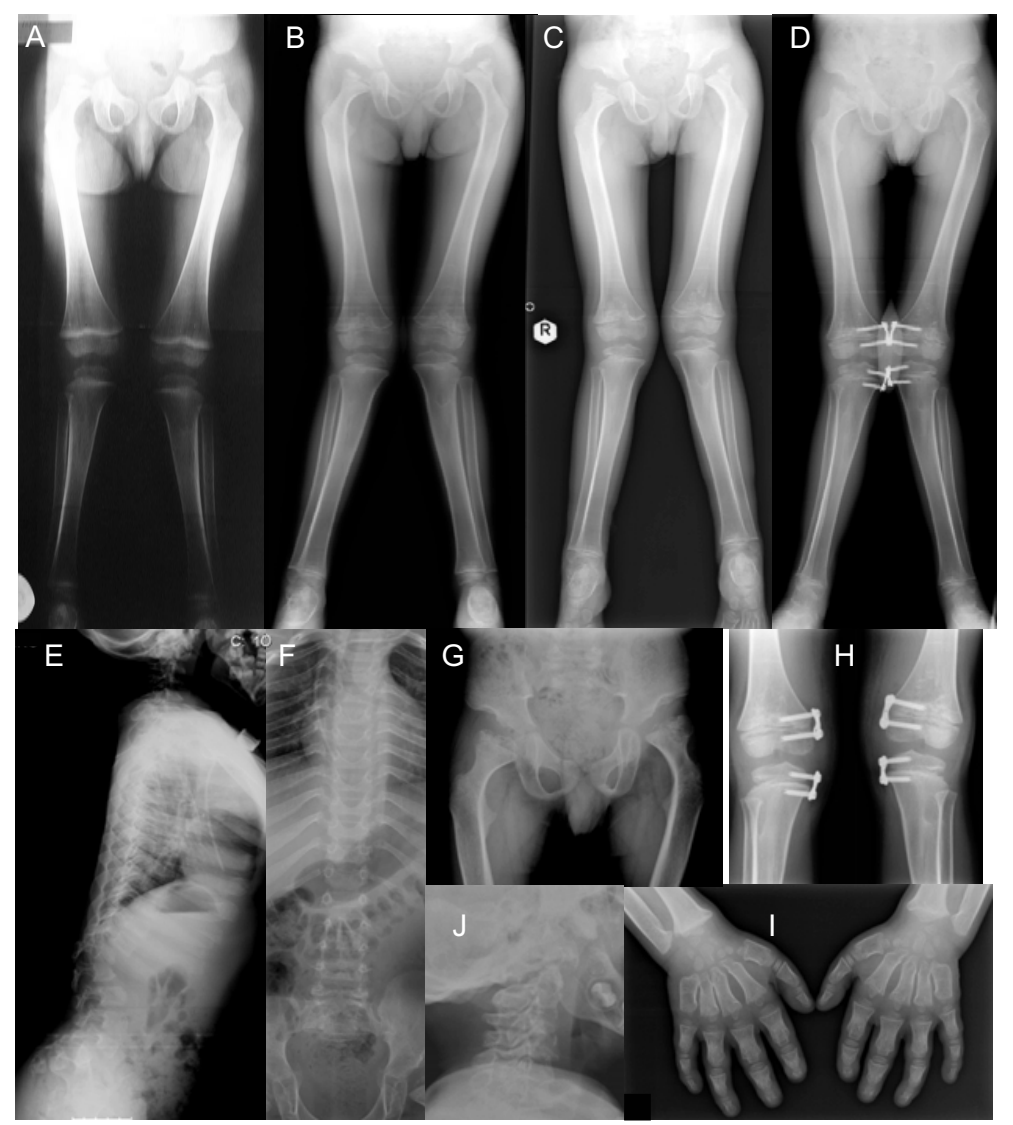

Fig. 2. X-ray films of Patient 2: lower limbs at 3.11 years (A), 7.3 years (B), 8.4 years (C), 9.2 years (D); spine at 9.2 years (E, F); hip at 9.2 years $(\mathrm{G})$; knees at 8.10 years $(\mathrm{H})$; hands at 8.10 years $(\mathrm{I})$.; and cervical spine at 8 years (J). Notice the typical dysostosis multiplex skeletal abnormalities at the spine, hip, long bones and hands. There is progressive hip disease and a bilateral genu valgum of $25^{\circ}$ (A-D) that recently has been addressed (D).

bar gibbus and bilateral inguinal hernias were noticed. The inguinal hernias were surgically corrected at 15 months. He had frequent respiratory infections and slight hepatosplenomegaly and pectus excavatum (hollowed chest). Follow-up evaluations disclosed discrete corneal opacity and a mild hearing defect. MPS VI diagnosis was confirmed by measuring ARSB activity in leucocytes at the age of 7 months. Mutation analysis showed compound heterozygosity for mutations c.1533_1555del and c.1336G >A; p.G446S (new mutation) in the $A R S B$ gene. At the age of 13 months, ERT with galsulfase ( $2 \mathrm{mg} / \mathrm{kg} /$ week) was initiated in the framework of a clinical trial.

Height and weight remain currently stable at the 95th and 75 th percentile, respectively.

This patient's main problem is kyphoscoliosis. From the beginning he had severe hypoplasia of the L2L3 vertebral bodies. During the second year of life, there was a striking progression of his spinal deformi- ty (Fig. 3 A-I). Thoracolumbosacral bracing was introduced at 2 years. There was relative stabilization of the lumbar kyphosis but a progression of the scoliosis. Mild abnormalities are seen at the level of the cervical spine (Fig. 3 J). No other significant extra-spinal complications have been reported thus far (Fig. 3. K-O). Currently, there is discussion on the future management of this patient's orthopedic problems (orthotic versus surgical spine treatment).

\subsubsection{Discussion}

This case demonstrates a very classic thoracolumbar kyphosis seen in MPS. It is not unusual to see the fierce progression of the deformity that is demonstrated here. The main points of discussion on the future management of this patient are that these deformities may progress at a very young age, but that treatment may be deferred. There is a limit, however, to how long one can wait to intervene. This child has a near nor- 


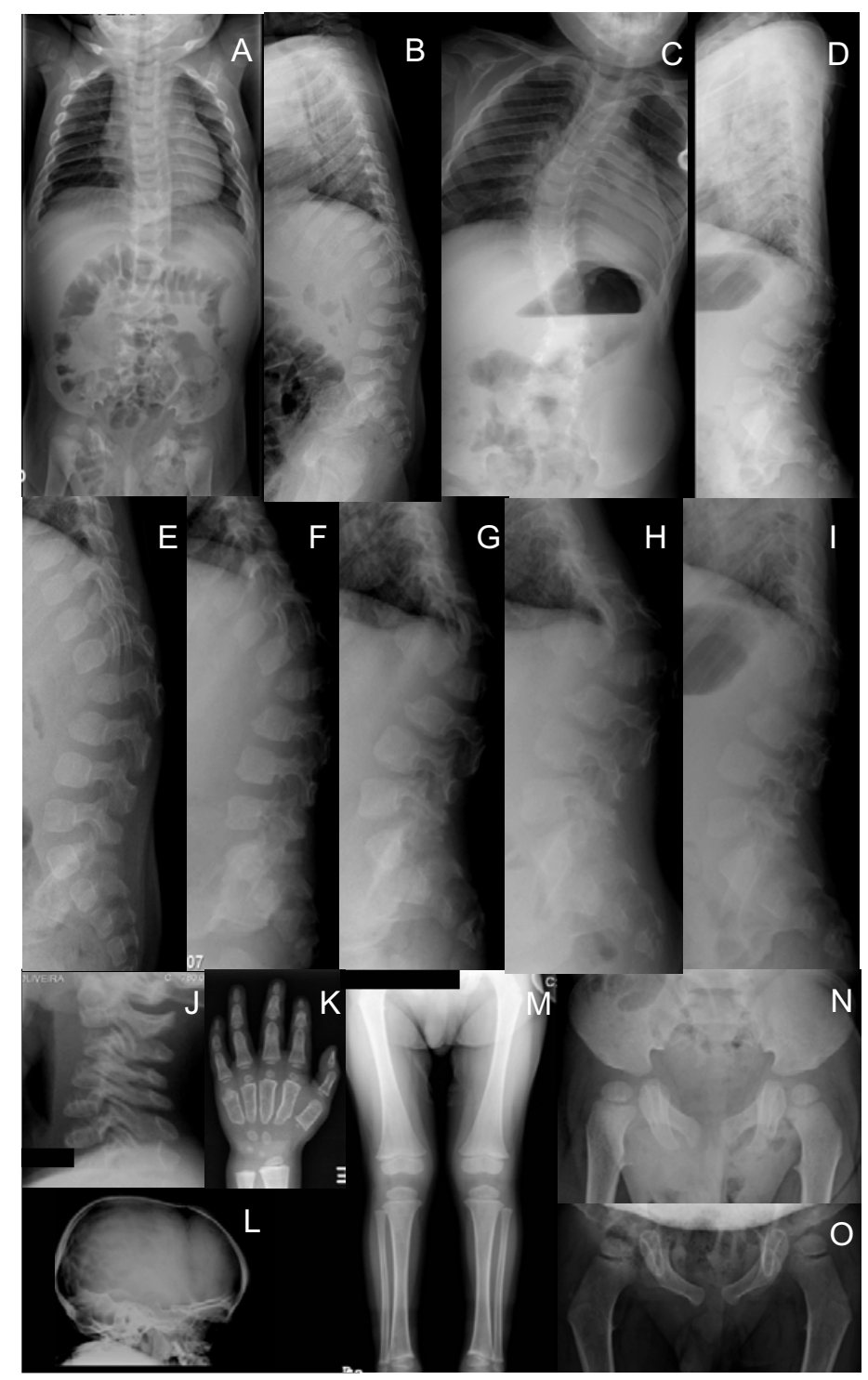

Fig. 3. X-ray films of Patient 3: Spine radiographs at 5 months (A,B) and 2.5 years (C,D), with Cobb angle of $30^{\circ}$; Lateral radiograph of the lumbar spine at 5 months $(\mathrm{E}), 1.1$ years $(\mathrm{F}), 1.8$ years $(\mathrm{G}), 1.11$ years $(\mathrm{H})$, and 2.5 years $(\mathrm{I})$ - Note the progression of the kyphosis. Bracing has been used since 2 years, with relative stabilization -; Lateral cervical spine at 1.5 years $(\mathrm{J})$; left hand at 2.1 years $(\mathrm{K})$; lateral cranium at 2.1 years $(\mathrm{L})$; lower limbs at 2.1 years $(\mathrm{M})$; and hip at 1.1 years $(\mathrm{N})$ and 2.1 years $(\mathrm{O})$. Notice the typical dysostosis multiplex abnormalities, without significant extra-spinal complications thus far.

mal growth velocity, which may have contributed to the aggressive deformity progression seen here. Because brace treatment of spinal deformities in MPS disorders has not, in general, been rigorously proven to be effective, it should only be used in exceptional cases. Surgical treatment for progressive thoracolumbar kyphosis should be strongly considered when the angular deformity exceeds $70^{\circ}$, if there is neurological compromise, or when associated pain becomes functionally limiting. An anterior and posterior fusion, with instrumentation (when possible) is the most reliable intervention. Postoperative bracing or casting may be required for up to six months after surgery.

\section{General discussion}

The above-described cases clearly illustrate that MPS VI can lead to significant skeletal deformities that require careful surveillance. As a result of bone and 
joint abnormalities, MPS VI patients often develop hip dysplasia, genu valgum, growth retardation, and gross abnormalities of the spine such as kyphosis or scoliosis [7]. Appropriate treatment of orthopedic problems can decrease the patients' morbidity and improve their quality of life considerably.

Several complications, such as spinal cord or carpal tunnel compression, which require awareness and timely intervention, may develop secondary to bone disease [18]. Clinical examination and imaging techniques should be used on a regular basis to follow these patients and detect complications of skeletal disease as early as possible. In fact, carpal tunnel compression of the median nerve and triggering of the flexor tendons is a situation that is often overlooked due to unusual absence of typical symptom of hand numbness or tingling and the difficulty in obtaining a detailed neurological exam including nerve conduction studies in the patients' age group. If treatment for triggering or median nerve compression is delayed, permanent damage to the median nerve and fixed contractures of the distal interphalangeal joints may occur. Besides carpal tunnel syndrome, cervical spine complications are an important issue. In addition to the possibility of cervical instability due to odontoid hypoplasia, GAG storage, inflammation, and fibrosis in the posterior longitudinal ligament and in dura (pachymeningitis cervicali) can cause compression of the spinal cord, particularly at the C1-C2 level. Ultimately, patients can become wheelchair-bound. Neurological assessment and a sagittal magnetic resonance imaging of the spine can monitor for this complication and suggest intervention before cervical myelopathy occurs.

All three patients are currently being treated with ERT (galsulfase). Galsulfase has been shown to increase endurance in a 12-min walk test and 3-min stair climb test in phase 2 and 3 clinical trials $[8,9,11]$. Secondary analyses of the galsulfase clinical studies and case reports have suggested that ERT can improve joint mobility $[8,10,16]$. As bone deformities are irreversible and often start to develop very early in life in MPS VI patients, early onset of ERT might be indicated in order to delay the development of severe skeletal dysplasia. Studies in cats have shown that ERT has a positive impact on bone development, particularly when started early in life $[1,4,6]$. So far, no studies on the impact of ERT on bone development in humans have been completed. Additional data are expected from several studies in sibling pairs and a clinical study in infants that are currently ongoing.

\section{Conclusions}

Orthopedic problems are common in MPS VI patients and can be extremely disabling. Early detection and management of these problems are warranted and can significantly improve the quality of life of these patients.

\section{Acknowledgements}

This manuscript was developed as the result of a meeting of experts entitled "Promoting Bone Health in MPS VI: Framing New Therapies" held in Oakland, California in October, 2008. Editorial assistance in the development of this manuscript was provided by Ismar Healthcare NV, Belgium, and was paid for by BioMarin Pharmaceutical Inc., Novato, CA. BioMarin reviewed the manuscript to insure the accuracy of all statements regarding enzyme replacement therapy with galsulfase, but had no role in the content presented and discussed at the meeting. All authors participated in the development and writing of the manuscript and are fully responsible for its content.

\section{Conflict of interest}

Klane White has received honoraria for educational lectures from BioMarin Pharmaceutical Inc., and Shire plc.

\section{References}

[1] D. Auclair, J.J. Hopwood, D.A. Brooks, J.F. Lemontt and A.C. Crawley, Replacement therapy in Mucopolysaccharidosis type VI: advantages of early onset of therapy, Mol Genet Metab 78 (2003), 163-174.

[2] P.J. Belmont, Jr. and D.W. Polly, Jr., Early diagnosis of Hurler's syndrome with the aid of the identification of the characteristic gibbus deformity, Mil Med 163 (1998), 711-714.

[3] W.P. Blount and G.R. Clarke, Control of bone growth by epiphyseal stapling: a preliminary report, J Bone Joint Surg Am 31A (1949), 464-478.

[4] S. Byers, J.D. Nuttall, A.C. Crawley, J.J. Hopwood, K. Smith and N.L. Fazzalari, Effect of enzyme replacement therapy on bone formation in a feline model of mucopolysaccharidosis type VI, Bone 21 (1997), 425-431.

[5] M.A. Cleary and J.E. Wraith, The presenting features of mucopolysaccharidosis type IH (Hurler syndrome), Acta Paediatr 84 (1995), 337-339.

[6] A.C. Crawley, K.H. Niedzielski, E.L. Isaac, R.C.A. Davey, S. Byers and J.J. Hopwood, Enzyme replacement therapy from birth in a feline model of mucopolysaccharidosis type VI, $J$ Clin Invest 99 (1997), 651-662. 
[7] R. Giugliani, P. Harmatz and J.E. Wraith, Management guidelines for mucopolysaccharidosis VI, Pediatrics 120 (2007), $405-418$.

[8] P. Harmatz, C.B. Whitley, L. Waber, R. Pais, R. Steiner, B. Plecko, P. Kaplan, J. Simon, E. Butensky and J.J. Hopwood, Enzyme replacement therapy in mucopolysaccharidosis VI (Maroteaux-Lamy syndrome), J Pediatr 144 (2004), 574-580.

[9] P. Harmatz, C.B. Whitley, L. Waber, R. Pais, R. Steiner, B. Plecko, P. Kaplan, J. Simon, J. Waterson and J.J. Hopwood, A phase I/II study of enzyme replacement therapy (ERT) for mucoploysaccharidosis VI (MPS VI; maroteaux-lamy syndrome): 48 week progress report, Am J Hum Genet 71 (2002), 582.

[10] P. Harmatz, D. Ketteridge, R. Giugliani, N. Guffon, E.L. Teles, M.C. Miranda, Z.F. Yu, S.J. Swiedler and J.J. Hopwood, Direct comparison of measures of endurance, mobility, and joint function during enzyme-replacement therapy of mucopolysaccharidosis VI (Maroteaux-Lamy syndrome): results after 48 weeks in a phase 2 open-label clinical study of recombinant human $\mathrm{N}$-acetylgalactosamine 4-sulfatase, Pediatrics 115 (2005), e681-e689.

[11] P. Harmatz, R. Giugliani, I. Schwartz, N. Guffon, E.L. Teles, M.C. Sá Miranda, J.E. Wraith, M. Beck, L. Arash, M. Scarpa, Z.F. Yu, J. Wittes, K.I. Berger, M.S. Newman and A.M. Lowe, Enzyme replacement therapy for mucopolysaccharidosis VI: a phase 3, randomized, double-blind, placebo-controlled, multinational study of recombinant human $\mathrm{N}$-acetylgalactosamine 4-sulfatase (recombinant human arylsulfatase B or rhASB) and follow-on, open-label extension study, J Pediatr 148 (2006), 533-539.
[12] R. Lachman, K.W. Martin, S. Castro, M.A. Basto, A. Adams and E. Leão Teles, Radiologic and neuroradiologic findings in the mucopolysaccharidoses, Journal of Pediatric Rehabilitation Medicine, Submitted (reference to another paper of these proceedings).

[13] E.F. Neufeld and J. Muenzer, The mucopolysaccharidoses, in: The Metabolic and Molecular Bases of Inherited Disease, C.R. Scriver, A.L. Beaudet, W.S. Sly and D. Valle, eds, McGrawHill Medical Publishing Division, New York, 2001, pp. 34213452.

[14] E. Odunusi, C. Peters, W. Krivit and J. Ogilvie, Genu valgum deformity in Hurler syndrome after hematopoietic stem cell transplantation: correction by surgical intervention, J Pediatr Orthop 19 (1999), 270-274.

[15] M.F.G. Petry, T. Dieter, M. Burin, R. Giugliani and S. Leistner, Identification of a novel mutation in the ARSB gene that is frequent among Brazilian MPSVI patients, Genet Test 7 (2003), 347-349.

[16] M. Scarpa, R. Barone, A. Fiumara, L. Astarita, G. Parenti, A. Rampazzo, S. Sala, G. Sorge and R. Parini, Mucopolysaccharidosis VI: the Italian experience, Eur J Pediatr 168 (2009), 1203-1206.

[17] P.M. Stevens, Guided growth for angular correction: a preliminary series using a tension band plate, J Pediatr Orthop 27 (2007), 253-259.

[18] J.A. Thorne, M. Javadpour, D.G. Hughes, E. Wraith and R.A. Cowie, Craniovertebral abnormalities in Type VI mucopolysaccharidosis (Maroteaux-Lamy syndrome), Neurosurgery 48 (2001), 849-852. 